\title{
Retrovirus Capsid Assembly and Polymorphism Studied by Cryo-EM and Cryo-ET.
}

\author{
G. Cardone*, N. Cheng*, J.B. Heymann*. C. Butan*, D.C. Winkler*, J.G. Purdy**, R.C. Craven** \\ and A.C. Steven* \\ * Laboratory of Structural Biology, National Institute for Arthritis, Musculoskeletal and Skin \\ Diseases, National Institutes of Health, Bethesda MD 20892; \\ ** Department of Microbiology and Immunology, The Pennsylvania State University College of \\ Medicine, Hershey PA 17033.
}

\begin{abstract}
Alpha-retroviruses, such as human immunodeficiency virus (HIV) and the avian pathogen, Rous sarcoma virus (RSV), have an external envelope surrounding the viral core - consisting of the capsid enclosing the diploid ssRNA genome and the replication enzymes - as well as a population of unassembled copies of capsid protein (CA). CA is incorporated into the immature virion as part of the Gag polyprotein from which it is released by the viral protease as the virion matures. A correctly formed core is thought to be essential for infectivity. However, investigation is hampered by capsid polymorphism. HIV capsids are mostly asymmetric cones, themselves polymorphic, while those of RSV are various irregular polyhedra or tubes. We have approached this problem by using cryoelectron tomography of isolated virions to visualize RSV capsids in situ, and cryo-EM and image reconstruction to study the structures of small icosahedral capsids assembled in vitro.
\end{abstract}

Cryo-ET of isolated virions was performed at $120 \mathrm{keV}$ [1], using an FEI Tecnai-12 equipped with an energy filter (Gatan) to collect tilt series at $1^{\circ}$ steps, using SerialEM. Projections were aligned using fiducial gold particles. Tomograms were reconstructed using IMOD and denoised by a nonlinear anisotropic diffusion algorithm implemented in Bsoft. Cryo-EM was performed on capsids assembled from recombinant CA protein. Focal image pairs of vitrified specimens were recorded on a CM200-FEG (FEI), operated at $120 \mathrm{keV}$. Reconstructions were performed by projection-matching with CTF-corrected data, starting from a model calculated from a single averaged 3-fold projection. Pseudo-atomic models of CA pentamer and hexamer were constructed by fitting separately atomic models from the PDB for the $\mathrm{N}$ - and $\mathrm{C}$-terminal domains of CA separately into capsid reconstructions. Fullerene constructions were generated using a custom module written in Chimera.

By cryo-ET, we documented three classes of intraviral RSV capsids: irregular polyhedra; closed capsids without sharply defined vertices, and tubes (often empty); $\sim 20 \%$ of virions have no capsids [1]. We found by model-building that the shapes of the polyhedral capsids can be explained as fullerene lattices based on 3-dimensional foldings of a hexagonal net with twelve 5-fold vertices, variously distributed ([1, 2]; Fig 1). It had been a matter of debate whether CA can form pentamers as the fullerene theory requires - in addition to the well documented hexamers. Our cryo-EM analysis of in vitro-assembled capsids demonstrated that this is indeed the case [3]. We observed $17 \mathrm{~nm}$ capsids made of 12 pentamers, and 30nm capsids made of 12 pentamers plus 20 hexamers. The latter capsids confirm that pentamers and hexamers can co-exist in the same shell. The polyhedral intraviral capsids visualized in tomograms have vertex sites that are not empty; we envisage that they are occupied by CA pentamers. Our working hypothesis is that a viable core is a closed shell of any morphology into which the viral ribonucleoprotein has been successfully packaged. 
References

[1] C. Butan et al., J. Mol. Biol. 376 (2008) 1168.

[2] G. Cardone et al, Nature 457(2009) 694.

[3] J.B. Heymann et al., Comp. \& Math. Meth. Med. 9, (2008) 197.

[4] This research was supported by the Intramural Research Program of NIAMS, the NIH IATAP program (ACS), and NIH grant CA100322 and the Pennsylvania Department of Health (RCC).


FIG. 1 Top left: Central section through a cryo-electron tomogram of an RSV virion. Top right: fullerene model of enclosed capsid. Bottom: A fullerene model (blue lattice) represents the capsid as a hollow shell of hexagons, completed by twelve pentagons. At each pentagonal vertex is a pentamer of N-terminal domains of CA, as determined by cryo-EM. In the background is a cryo-micrograph of icosahedral capsids (density inverted). 